\title{
Influencia de los valores periodísticos y empresariales en la reputación de los medios de comunicación en España
} Influence of journalistic and business values on the
reputation of the media in Spain

Yolanda Ortiz-de-Guinea-Ayala; Juan-José Nájera-Sánchez; Antonio Montero-Navarro

Cómo citar este artículo:

Ortiz-de-Guinea-Ayala, Yolanda; Nájera-Sánchez, Juan-José; Montero-Navarro, Antonio (2020). "Influencia de los valores periodísticos y empresariales en la reputación de los medios de comunicación en España". El profesional de la información, v. 29, n. 3, e290317.

https://doi.org/10.3145/epi.2020.may.17

Artículo recibido el 26-07-2019

Aceptación definitiva: 16-12-2019

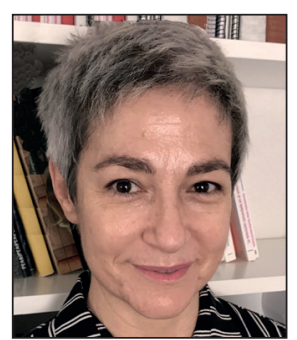

Yolanda Ortiz-de-Guinea-Ayala $\triangle$ https://orcid.org/0000-0001-8560-656X

Universidad Rey Juan Carlos Dto. de Comunicación y Sociología Camino del Molino, s/n. 28943 Fuenlabrada (Madrid), España yolanda.ortizdeguinea.ayala@urjc.es

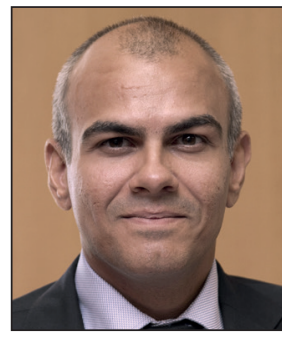

Juan-José Nájera-Sánchez https://orcid.org/0000-0003-1012-6136

Universidad Rey Juan Carlos Dto. de Economía de la Empresa (ADO) Paseo de los Artilleros, $\mathrm{s} / \mathrm{n}$. 28032 Madrid, España juanjose.najera@urjc.es

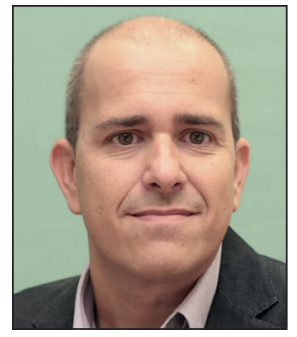

\author{
Antonio Montero-Navarro \\ https://orcid.org/0000-0001-8096-5352 \\ Universidad Rey Juan Carlos \\ Dto. de Economía de la Empresa (ADO) \\ Paseo de los Artilleros, s/n. \\ 28032 Madrid, España \\ antonio.montero@urjc.es
}

\section{Resumen}

El estudio de la reputación ha cobrado importancia en los últimos años aplicado a diversos ámbitos (empresas, territorios, instituciones...), como demuestra el creciente volumen de publicaciones científicas de este tema. Este trabajo analiza de forma específica la reputación de los medios de comunicación (prensa, tanto en papel como digital, radio y televisión), aportando por primera vez una evidencia empírica acerca de los valores canónicos de los que depende la reputación mediática. Asimismo, se analizan las variables en las que los valores se desagregan y que concretan cualitativa y cuantitativamente esa dependencia. Gracias al análisis multivariable de los resultados de una encuesta realizada en España entre más de un centenar de profesionales y gestores de una muestra de medios de comunicación, se ha podido establecer que los valores periodísticos o profesionales suponen aproximadamente las tres cuartas partes de su reputación y que el resto corresponde al segundo de los valores canónicos de la reputación mediática: los valores empresariales.

\section{Financiación}

Esta investigación se encuadra dentro de otra más amplia que tiene como objeto evaluar empíricamente la reputación de los medios de comunicación en España y en Europa, que ha sido parcialmente financiada por el Mineco (Ministerio de Economía, Industria y Competitividad) en su convocatoria del Programa Estatal de I+D+i de 2017, orientado a los Retos de la sociedad. 


\title{
Palabras clave
}

Reputación mediática; Valores de reputación mediática; Variables de reputación mediática; Universo mediático; Medios de comunicación; Valores periodísticos; Valores empresariales; Análisis factorial; Análisis multivariable.

\begin{abstract}
The study of reputation, applied to different objects (companies, territories, institutions...) has gained relevance in the last years, as it can be seen from the increasing volume of scientific papers dealing with the subject. This paper analyzes the corporate reputation of media enterprises (press, radio and television), providing for the first time an empirical evidence about the canonical values on which the media reputation depends. Likewise, the variables in which the values are disaggregated and which qualitatively and quantitatively define this dependency are analyzed. Thanks to the multivariate analysis of the results of a survey conducted in Spain among more than a hundred professionals and managers who work for a sample of media, it has been possible to establish that the journalistic or professional values account for approximately three quarters of their reputation and that the rest corresponds to the second of the canonical values of the media reputation: the business values.
\end{abstract}

\section{Keywords}

Media reputation; Values of media reputation; Media reputation variables; Media universe; Media; Journalistic values; Business values; Factor analysis; Multivariate analysis.

\section{Introducción}

Mediante este trabajo se ha testado una metodología diseñada por Ortiz-de-Guinea-Ayala, Villafañe y Caffarel-Serra (2018). El texto actual recoge el inicio del proceso metodológico que se ensayó en el contexto mediático de la provincia de Castellón en 2017, planteando entonces como hipótesis el origen de la reputación de los medios de comunicación desde el punto de vista de la Teoría de la reputación: los valores periodísticos o profesionales y los valores empresariales. Ambos se constituyen en los valores canónicos que, entendidos como las dimensiones estructurales de la reputación mediática, sirven para explicarla (Ortiz-de-Guinea-Ayala; Villafañe; Caffarel-Serra, 2018).

El objetivo de esta investigación es múltiple. En primer lugar se ha realizado un recorrido por la Teoría de la reputación (Fombrun, 1996; Villafañe, 2013), con el fin de establecer desde un punto de vista teórico la naturaleza de los dos valores canónicos (profesionales y empresariales) que componen la reputación de un medio de comunicación.

\section{El origen de la reputación de los medios de comunicación desde el punto de vista de la Teoría de la reputación son los va- lores periodísticos o profesionales y los valores empresariales}

Un segundo objetivo consiste en la identificación de las variables que explican esos valores canónicos. Para ello, con posterioridad al estudio teórico se ha efectuado un análisis multivariable con el fin de determinar para el caso de los profesionales de los medios de información, si esas variables que la teoría y el estudio cualitativo sugieren se agrupan en esos dos valores canónicos. Además, se ha contrastado cuál es el poder explicativo conjunto que dichos valores tienen sobre la reputación y cuál es la importancia de cada uno de ellos por separado.

Esta aportación es de especial relevancia, ya que no existen precedentes ni teóricos ni empíricos en esta cuestión. La presente investigación constituye el primer estudio empírico sobre la reputación de los medios de comunicación. Este trabajo pretende contribuir a la consolidación de una propuesta de una Teoría de la Reputación de los Medios de Comunicación epistemológicamente autónoma, aunque encuadrada en el marco teórico específico de la Teoría de la Reputación Corporativa y en el más genérico de las Ciencias Sociales.

\section{Marco teórico}

Cuando pensamos en la reputación es frecuente asociar este concepto con una valoración positiva y favorable por parte de una serie de agentes que hace que las relaciones con éstos puedan ser más fluidas. Si una persona por ejemplo tiene una buena reputación entre sus vecinos, confiarán en él o ella y esto le permitirá poder solicitarles cosas en base a dicha confianza.

Esta noción de reputación personal se ha extendido a las empresas, es la denominada reputación corporativa. Fombrun propone una de las definiciones más citadas y utilizadas, que caracteriza a la reputación corporativa como

"la representación de las percepciones sobre las acciones pasadas de una empresa y sus perspectivas de futuro que describen de forma global el atractivo de ésta para sus principales grupos de interés en comparación con otros rivales destacados" (Fombrun, 1996, p. 165).

Villafañe rechaza el término percepción que asocia exclusivamente a la imagen, justificando que se trata de nociones diferentes ya que la reputación -afirma este autor- se basa en la realidad, y define la reputación corporativa como el 
reconocimiento de la realidad y del comportamiento corporativo que hacen los stakeholders de una empresa de ese comportamiento empresarial en función de su grado de satisfacción de las expectativas (Villafañe, 2013).

Por su parte, Carreras, Alloza y Carreras definen la reputación corporativa como un

"conjunto de evaluaciones colectivas, suscitadas por el comportamiento corporativo en las distintas audiencias, que motivan sus conductas de apoyo u oposición y que son capaces de generar valor corporativo" (Carreras; Alloza; Carreras, 2013).

Los autores contemplan tanto la relevancia de las acciones de la empresa como las valoraciones de las mismas realizadas por los grupos de interés.

De este modo, al igual que sucede con un individuo, la reputación de una empresa es el balance de las valoraciones que tienen sobre ella diversos agentes. En esta definición podemos ver que la reputación corporativa deriva de las acciones del pasado y del presente de una empresa, y marca las expectativas de los grupos de interés que se relacionan con ella.

Cabe resaltar dos aspectos adicionales en relación con la reputación corporativa. En primer lugar, al igual que sucede con un individuo, una buena reputación proporciona a una empresa una serie de ventajas:

- sus productos (y sus acciones) son más atractivos;

- la compañía es considerada un empleador más deseable, lo que facilita su acceso favorable al mejor capital humano (Fombrun, 1996);

- se reducen sus costes de transacción con proveedores y clientes, dada su fiabilidad (De-la-Fuente-Sabaté; Quevedo-de-Puente, 2003);

- soporta mejor los embates de una posible crisis económica (Bhattacharya; Sen, 2004);

- favorece la evolución desde organizaciones satisfechas a organizaciones comprometidas en su aplicación al ámbito de la dirección de personas y la gestión del talento en las organizaciones (Villafañe, 2017).

Siguiendo a Chun (2005), podemos considerar tres grandes escuelas de pensamiento en el estudio de la reputación, lo que indudablemente influye en las definiciones y formas de medir esta variable. La autora distingue:

- una perspectiva evaluativa, desde la que la reputación es un recurso valioso (Grant, 2006) como potencial fuente de ventaja competitiva (Hall, 1993), muy vinculada con la dirección estratégica de la empresa y cercana a los rankings de reputación, especialmente al más conocido de ellos, la metodología Fortune Most Admired Company, apoyados generalmente en la visión de un único stakeholder;

- una visión impresional, fundamentada en las impresiones que mantienen sobre la empresa determinados grupos de interés, especialmente sus empleados (Gioia; Thomas, 1996) o sus clientes (Bromley, 1993);

- una escuela relacional, que trata de analizar la vinculación entre las perspectivas interna y externa de la organización, valorando las similitudes y diferencias entre la percepción interna (identidad) y externa (imagen) de una compañía (Davies; Chun, 2002; Chun; Davies, 2006).

Desde el punto de vista de la economía de la empresa, el origen epistemológico del estudio de la reputación corporativa es múltiple, pudiendo ser abordado desde diferentes perspectivas complementarias y no excluyentes. Por una parte, teniendo en cuenta que la reputación de una empresa se asienta en su relación con los diferentes grupos de interés o stakeholders, una primera aproximación a su estudio se da desde la visión de Jensen y Meckling (1976) de la empresa como nexo de contratos. Como plantean De-la-Fuente-Sabaté y Quevedo-de-Puente (2003), la reputación supone no sólo una señal informativa, sino además una garantía contractual para los diferentes agentes que interactúan con la empresa, reduciendo de este modo los costes de transacción de la contraparte (Williamson, 1975). De este modo, el coste de trabajar para asociarse con, o ser proveedor de una empresa con buena reputación, es menor que el de hacerlo con una empresa desconocida, y obviamente que el de hacerlo con una organización valorada negativamente por los diferentes grupos de interés.

La reputación ha sido analizada como un recurso, posiblemente el intangible más valioso de una empresa

(Martín-de-Castro; Navas-López; López-Sáez; 2006; Cravens; Goad-Oliver; Ramamoorti, 2003; Reuber; Fis-

\section{La presente investigación constituye el primer estudio empírico sobre la reputa- ción de los medios de comunicación} cher, 2005). La reputación de una empresa la diferencia de otras organizaciones (Shamsie, 2003), contribuyendo de forma decisiva a la creación de valor de la empresa (Boyd; Bergh; Ketchen, 2010). Como recurso, su gestión es tarea de los directivos, cuidando de su acumulación a lo largo del tiempo y poniendo en marcha las acciones necesarias para evitar su posible deterioro.

Como último fundamento epistemológico, podemos relacionar la noción de reputación con la visión planteada por Nahapiet y Ghoshal del concepto de capital social, que consiste en

"la suma de los recursos actuales y potenciales incluidos, disponibles o derivados de la red de relaciones que posee un individuo o una unidad social” (Nahapiet; Ghoshal, 1998)

que en el caso que nos ocupa, podría ser una organización. 
Arregle et al. (2007) definen el capital social de una empresa como las relaciones entre los individuos y las organizaciones que facilitan la actuación y crean valor. Los beneficios del capital social de una empresa son en buena medida los mismos que cabe esperar de una buena reputación corporativa (Fombrun, 1996):

- contribuye a la creación de valor (Arregle et al., 2007);

- reduce los costes de transacción vinculados a la búsqueda, ajuste y refuerzo de los contratos (Gulati, 1998);

- reduce el coste de acceso a los diferentes mercados (Williamson, 1975).

De este modo el estudio de la reputación corporativa se asienta también en la Teoría del capital social.

Las definiciones proporcionadas anteriormente distan de ser aceptadas de forma unánime. De hecho, como indican Lange, Lee y Dai (2011), el estudio de la reputación corporativa se podría situar en una fase protocientífica, en la que no hay de hecho un consenso universal en torno a la caracterización del fenómeno estudiado. En su trabajo, estos autores analizan las diferentes definiciones proporcionadas por otros artículos, apuntando a la existencia de tres grandes grupos de trabajos.

El primero de ellos es denominado por los autores como being known, literalmente "ser conocido". Podemos incluir en este primer conjunto artículos como el de Rindova et al. (2005), que habla de prominence; o el de Barnett, Jermier y Lafferty (2006) que utilizan el término awareness. Quizás la traducción más precisa al castellano sería notoriedad. Para Lange, Lee y Dai (2011), los trabajos incluidos en este grupo no tratan de emitir un juicio de valor sobre la empresa, sino de posicionarla comparativamente respecto a sus competidores o rivales.

El segundo conjunto de trabajos es caracterizado por los autores como being known for something, lo que se traduciría literalmente como "ser conocido por algo", aunque el término que mejor se adaptaría sería el de reconocimiento. Rindova et al. hablan del

"grado en que los stakeholders valoran positivamente a una organización en un determinado atributo, como por ejemplo su capacidad para producir bienes de calidad" (Rindova et al., 2005, p. 7).

La definición proporcionada por Fischer y Reuber (2007) es muy similar, cuando consideran que la reputación es la valoración de una característica concreta, ya sea la mencionada capacidad de producir bienes de calidad o la de competir ventajosamente en precios. Lange, Lee y Dai (2011) consideran que estas definiciones de reputación sí se basan en una evaluación, en una valoración positiva por parte de un agente externo.

El tercer gran grupo de trabajos es denominado por los autores generalized favorability, literalmente "favorabilidad generalizada", aunque podría condensarse en el término atractivo. Barnett, Jermier y Lafferty (2006) hablan de la estima, el aprecio hacia la firma o el grado de atractivo que esta tiene. Los mismos autores mencionan los juicios colectivos de una organización por parte de los observadores, basados en su impacto económico, social y ambiental a lo largo del tiempo. Estos trabajos presentan una mirada global, que descansa en un conjunto de grupos de interés que comparan a la empresa con otras referencias.

Los medios de comunicación no están exentos de su evaluación reputacional. Se trata de organizaciones valoradas por un conjunto de agentes, tanto externos como internos. La opinión que estos tengan se forja a lo largo del tiempo, y determinadas actuaciones puntuales pueden dañar de forma dramática dicha valoración. Se han propuesto indicadores de responsabilidad social y reputación corporativa para medir la confianza de las audiencias en las marcas televisivas, centrados en los programas de entretenimiento televisivo (Morales-Blanco-Steger; Fuente-Cobo, 2018). Finalmente, del mismo modo que sucede en otros ámbitos empresariales, una reputación favorable se plasma en determinadas ventajas para el medio, como puede ser una mejor valoración por parte de sus seguidores actuales y potenciales o un mayor atractivo de cara a conseguir la colaboración de diferentes profesionales. Por tanto, evaluar y gestionar la reputación de un medio de comunicación debe ser, al igual que en cualquier organización, una de las tareas más importantes para su dirección (Cravens; Goad-Oliver; Ramamoorti, 2003).

Este trabajo está orientado a conocer cuáles son los factores que determinan la reputación de un medio de comunicación. De este modo, si consideramos los tres grandes bloques de estudios relacionados con la definición de la reputación corporativa, nos situamos más cercanos al segundo, que habla del reconocimiento. Tratamos, siguiendo a Rindova et al. (2005), de conocer el grado en que los grupos de interés valoran a un medio en un determinado atributo o, en este caso, en un conjunto limitado de atributos.

El trabajo de Ortiz-de-Guinea-Ayala, Villafañe y Caffarel-Serra (2018) en relación con los medios de comunicación en la provincia de Castellón, realiza una primera aproximación a cuáles pueden ser estos valores y atributos. Para ello consulta a diferentes grupos de expertos (periodistas, directores de comunicación en empresas, directivos de medios, políticos, empresarios y, finalmente, académicos y líderes de opinión), que identifican la existencia de dos grandes valores canónicos que explican la reputación de un medio de comunicación: los valores periodísticos o profesionales y Los medios de comunicación no están exentos de su evaluación reputacional 
riza por un conjunto de atributos específicos, que servirán como punto de partida en este estudio. En dicho trabajo se concluye que los valores periodísticos tendrían un peso superior a los empresariales a la hora de determinar la reputación de dicho medio.

No obstante, como los propios autores señalan, se trata de una primera aproximación al estudio de la reputación corporativa de los medios de comunicación, que requiere un trabajo más profundo, apoyándose en una metodología cuantitativa. Partiendo por tanto de los resultados de dicho trabajo, y con el fin de alcanzar los objetivos citados en la introducción de este estudio, planteamos las siguientes hipótesis.

H1: Existen dos valores canónicos, los periodísticos y los empresariales, que aglutinan las principales variables relacionadas con la reputación de los medios de comunicación para los profesionales de la comunicación.

H2: Los valores canónicos periodísticos y empresariales explican la reputación de los medios de comunicación para los profesionales de la información.

H3: El peso del valor canónico periodístico es mayor que el del valor canónico empresarial en la explicación de la reputación de los medios de comunicación para los profesionales de la información.

\section{Metodología}

Como hemos podido ver, desde un punto de vista teórico existen dos tipos de valores que determinan la reputación de los medios de comunicación: los valores periodísticos o profesionales y los valores empresariales asociados a cada medio.

Con el fin de contrastar empíricamente la influencia de ambos tipos de valores sobre la citada reputación se efectuó en primer lugar un estudio cualitativo para seleccionar el universo mediático y lograr una muestra representativa del mismo y, en segundo lugar, se realizó un estudio cuantitativo para jerarquizar los dos valores de reputación mediática e identificar primero, y medir a continuación la influencia de las variables que los componen y que más influyen en su reputación.

Durante 2018 se invitó a participar a una veintena de directores de medios y agencias de noticias en un estudio cualitativo. Finalmente 12 de estos directivos (entre otros, pertenecientes a medios como $A B C$, El confidencial, Infolibre, Europa Press, Eldiario.es...) fueron entrevistados en esta fase. Este análisis cualitativo, entre otros interesantes resultados, nos permitió obtener el conjunto de medios objeto del análisis empírico del presente estudio. La muestra quedó configurada por los 16 medios que más consenso obtuvieron entre los directores entrevistados. Estos, alfabéticamente ordenados, son: $A B C$, Antena 3, COPE, El confidencial, Eldiario.es, El mundo, El país, El periódico de Cataluña, La vanguardia, La sexta, InfoLibre, Onda Cero, RNE, TVE, Cadena SER y Tele 5.

Del estudio cualitativo se obtuvo un repertorio muy amplio y heterogéneo de variables de reputación, tanto periodísticas como empresariales. Partiendo de estas variables y apoyados en el estudio previo de Ortiz-de-Guinea-Ayala, Villafañe y Caffarel-Serra (2018), se solicitó a los entrevistados la selección y clasificación de estas variables para así configurar el cuestionario que posteriormente se ha utilizado con diversos públicos, si bien en este trabajo sólo hemos analizado la encuesta a profesionales del sector de la información.

Dado que no existe un censo único de profesionales y su número se considera suficientemente elevado, se optó por considerar que la población objeto de estudio es infinita. Debido a cuestiones prespuestarias, la muestra final fue de 135 casos, con un error muestral de $\pm 8,4 \%$. La encuesta se ha realizado mediante entrevista telefónica asistida por ordenador, utilizando el cuestionario incluido en el anexo. La encuesta fue encargada a una empresa dedicada a la investigación de mercados a la que se facilitaron los contactos de los diferentes medios de comunicación y de asociaciones profesionales españolas. Se solicitó que la muestra fuera aleatoria.

Cada encuestado era preguntado en primer lugar por su grado de conocimiento acerca de diferentes medios de comunicación: ocho de prensa ( $A B C, E l$ país, El mundo, La vanguardia, El periódico de Cataluña, El confidencial, Eldiario.es e Infolibre); cuatro de radio (COPE, Onda Cero, RNE y Cadena SER); y cuatro de televisión (Antena 3, La sexta, TVE y Tele 5). Con esta pregunta se trataba de determinar si el encuestado seguía habitualmente el medio, lo seguía esporádicamente, no lo seguía habitualmente, pero tenía referencias o bien no tenía prácticamente referencias de dicho medio. Posteriormente, cada encuestado pasaba a puntuar en sus valores y su reputación global únicamente a ocho medios, elegidos entre aquellos de los que tuviera al menos algunas referencias, si bien en su mayoría las valoraciones fueron emitidas por encuestados que seguían esporádica o habitualmente el medio valorado. En las tablas 1 y 2 se resumen los estadísticos descriptivos para las diferentes variables utilizadas para medir los valores canónicos de la reputación y la valoración subjetiva global de la reputación (considerando las valoraciones realizadas para todos los medios incluidos en la muestra), así como el grado de conocimiento de los informantes y número de valoraciones que se realizaron para cada medio. 
Tabla 1. Estadísticos descriptivos

\begin{tabular}{|c|c|c|}
\hline & \\
\hline & Media & Desviación típica \\
\hline 1. Credibilidad & 6,19 & 1,85 \\
\hline 2. Rigor informativo & 6,22 & 1,81 \\
\hline 3. Calidad periodística & 6,44 & 1,76 \\
\hline 4. Independencia & 5,36 & 2,12 \\
\hline 5. Ética y deontología profesional & 5,97 & 1,88 \\
\hline 6. Contribución a la democracia & 6,36 & 2,07 \\
\hline 7. Sostenibilidad e independencia económica & 5,58 & 2,00 \\
\hline 8. Transparencia en la gestión & 5,25 & 2,10 \\
\hline 9. Responsabilidad de la propiedad & 5,50 & 2,07 \\
\hline 10. Derechos y deberes de los trabajadores & 5,89 & 1,82 \\
\hline 11. Reputación global del medio & 6,61 & 6,57 \\
\hline
\end{tabular}

Tabla 2. Grado de conocimiento y número de valoraciones por medio

\begin{tabular}{|l|c|c|c|c|}
\hline \multicolumn{1}{|c|}{ Es un medio que... } & Sigo habitualmente & $\begin{array}{c}\text { Sigo esporádica- } \\
\text { mente }\end{array}$ & $\begin{array}{c}\text { No sigo, pero sí tengo } \\
\text { referencias }\end{array}$ & Respuestas finales \\
\hline ABC & $46,7 \%$ & $40,0 \%$ & $13,3 \%$ & 60 \\
\hline El país & $94,4 \%$ & $5,6 \%$ & $0,0 \%$ & 90 \\
\hline El mundo & $77,8 \%$ & $18,9 \%$ & $3,3 \%$ & 90 \\
\hline La vanguardia & $67,6 \%$ & $26,8 \%$ & $5,6 \%$ & 71 \\
\hline El periódico de Cataluña & $51,0 \%$ & $38,8 \%$ & $10,2 \%$ & 49 \\
\hline El confidencial & $65,8 \%$ & $30,1 \%$ & $4,1 \%$ & 73 \\
\hline Eldiario,es & $57,1 \%$ & $32,9 \%$ & $10,0 \%$ & 70 \\
\hline InfoLibre & $51,2 \%$ & $34,9 \%$ & $14,0 \%$ & 43 \\
\hline Cope & $44,4 \%$ & $29,6 \%$ & $25,9 \%$ & 54 \\
\hline Onda Cero & $53,4 \%$ & $24,1 \%$ & $22,4 \%$ & 58 \\
\hline RNE & $50,7 \%$ & $39,1 \%$ & $10,1 \%$ & 69 \\
\hline Cadena SER & $80,0 \%$ & $14,7 \%$ & $5,3 \%$ & 95 \\
\hline Antena 3 & $52,7 \%$ & $34,5 \%$ & $12,7 \%$ & 55 \\
\hline La sexta & $66,2 \%$ & $31,2 \%$ & $2,6 \%$ & 77 \\
\hline TVE & $82,9 \%$ & $14,6 \%$ & $2,4 \%$ & 82 \\
\hline Tele 5 & $38,6 \%$ & $54,5 \%$ & $6,8 \%$ & \\
\hline
\end{tabular}

Como puede apreciarse en la tabla 2, no se utilizaron en ningún caso informantes que al menos no tuvieran referencias claras del medio, maximizándose el número de respuestas recogidas por encuestados que tuvieran un mayor grado de conocimiento del medio.

Con el fin de verificar las hipótesis planteadas, se procedió a realizar un análisis factorial, una técnica cuyo propósito es precisamente la agrupación de un determinado conjunto de variables, cuyos valores conocemos para cada una de las observaciones, en un número menor de dimensiones o variables no correlacionadas entre sí, presentes de forma latente, a las que denominaremos factores. Las variables observadas en la investigación actúan como combinaciones lineales de los mencionados factores más un término de error. El uso de esta técnica permite aglutinar dichas variables en dimensiones internamente consistentes, que pueden a su vez ser utilizadas en posteriores análisis estadísticos. Dado que en nuestro caso esperamos que el estudio corrobore la existencia de dos factores cuya existencia cabe a priori esperar -los ya citados valores periodísticos o profesionales y los valores empresariales- nuestro análisis factorial tiene un carácter confirmatorio.

\section{Resultados}

Como se comentó en la introducción, los objetivos de este trabajo consisten, en primer lugar, en ratificar lo que indica la Teoría de la reputación, aplicada a los medios de comunicación que, como ya se ha dicho, establece dos valores canónicos -los periodísticos o profesionales y los empresariales- y, en segundo lugar, medir la influencia de dichos valores canónicos sobre la reputación de los medios de comunicación empíricamente evaluados.

Un primer paso en el mencionado análisis factorial es el cálculo de la matriz de correlaciones ${ }^{1}$, así como la determinación de la adecuación del análisis factorial a nuestro propósito. Dado que se persigue la agrupación de las variables en factores subyacentes, para que el análisis pueda arrojar resultados válidos se requiere la existencia de grupos de variables 
altamente correlacionados internamente, siendo de este modo indeseable que dicha matriz se aproxime a la identidad, en la que cada variable sólo estaría correlacionada consigo misma. Como podemos ver en la tabla 3, no parece ser ese el caso: existe una fuerte correlación entre las diferentes variables, lo que es un buen indicio acerca de la existencia de los citados factores subyacentes.

Tabla 3. Matriz de correlaciones entre los diferentes valores evaluados

\begin{tabular}{|c|c|c|c|c|c|c|c|c|c|c|}
\hline & & & & & & & & & & \\
\hline & 1 & 2 & 3 & 4 & 5 & 6 & 7 & 8 & 9 & 10 \\
\hline 1. Credibilidad & 1 & 0,895 & 0,831 & 0,701 & 0,809 & 0,781 & 0,453 & 0,618 & 0,654 & 0,593 \\
\hline 2. Rigor informativo & 0,895 & 1 & 0,851 & 0,702 & 0,821 & 0,776 & 0,458 & 0,603 & 0,642 & 0,609 \\
\hline 3. Calidad periodística & 0,831 & 0,851 & 1 & 0,611 & 0,782 & 0,734 & 0,374 & 0,577 & 0,586 & 0,580 \\
\hline 4. Independencia & 0,701 & 0,702 & 0,611 & 1 & 0,739 & 0,693 & 0,471 & 0,573 & 0,600 & 0,585 \\
\hline 5. Ética y deontología profesional & 0,809 & 0,821 & 0,782 & 0,739 & 1 & 0,798 & 0,453 & 0,650 & 0,669 & 0,652 \\
\hline 6. Contribución a la democracia & 0,781 & 0,776 & 0,734 & 0,693 & 0,798 & 1 & 0,425 & 0,582 & 0,619 & 0,628 \\
\hline 7. Sostenibilidad e independencia económica & 0,453 & 0,458 & 0,374 & 0,471 & 0,453 & 0,425 & 1 & 0,619 & 0,583 & 0,572 \\
\hline 8. Transparencia en la gestión & 0,618 & 0,603 & 0,577 & 0,573 & 0,650 & 0,582 & 0,619 & 1 & 0,737 & 0,647 \\
\hline 9. Responsabilidad de la propiedad & 0,654 & 0,642 & 0,586 & 0,600 & 0,669 & 0,619 & 0,583 & 0,737 & 1 & 0,625 \\
\hline 10. Derechos y deberes de los trabajadores & 0,593 & 0,609 & 0,580 & 0,585 & 0,652 & 0,628 & 0,572 & 0,647 & 0,625 & 1 \\
\hline
\end{tabular}

Existen diferentes medidas de la adecuación del análisis factorial a los datos disponibles y variables analizadas. La medida de adecuación muestral KMO (de Kaiser-Meyer-Olkin) compara el coeficiente de correlación simple entre dos variables con la suma de dicho coeficiente y la correlación parcial entre ellas (excluyendo el efecto del resto de variables), siendo deseables valores cercanos a la unidad. En el caso de nuestro estudio, el valor obtenido es altamente satisfactorio $(0,943)$. Por su parte, la prueba de esfericidad de Bartlett compara la matriz de correlaciones con una matriz identidad, no pudiendo garantizarse la adecuación del análisis factorial si su significación es superior a 0,05. Nuevamente, esta prueba garantiza la adecuación del análisis factorial en nuestro caso.

Las dos siguientes etapas del proceso consisten en la extracción de los factores, siguiendo el mencionado procedimiento de componentes principales en nuestro caso, y la rotación de estos. En el primer paso, dado que esperamos la existencia de dos factores, lo hemos indicado desde el principio. En el segundo se trata de ajustar los factores de forma que las variables tiendan a cargar de forma esencial en un único factor, siendo mucho menor su relación con el resto. En nuestro caso hemos utilizado la rotación Varimax, que tiende a reducir el número de variables con saturación alta en cada factor mientras que a su vez cada factor tiende a tener cargas muy altas o muy bajas en cada variable. Los resultados pueden observarse en la tabla 4.

Tabla 4. Factores resultantes del estudio

\begin{tabular}{|l|c|c|}
\multicolumn{1}{c|}{} & \multicolumn{1}{c}{\begin{tabular}{c} 
Factor 1 \\
\cline { 2 - 3 } \multicolumn{1}{c|}{}
\end{tabular}} & Vactor $\mathbf{2}$ \\
\hline Credibilidad & 0,873 & 0,329 \\
\hline Rigor informativo & 0,881 & 0,323 \\
\hline Calidad periodística & 0,873 & 0,246 \\
\hline Independencia & 0,692 & 0,428 \\
\hline Ética y deontología profesional & 0,830 & 0,392 \\
\hline Contribución a la democracia & 0,817 & 0,341 \\
\hline Sostenibilidad e independencia económica & 0,140 & 0,879 \\
\hline Transparencia en la gestión & 0,420 & 0,767 \\
\hline Responsabilidad de la propiedad & 0,484 & 0,707 \\
\hline Derechos y deberes de los trabajadores & 0,473 & 0,673 \\
\hline
\end{tabular}

Los resultados obtenidos confirman la existencia de los dos valores canónicos de la reputación mediática:

- los periodísticos o profesionales, que se corresponden con las seis primeras variables analizadas;

- los empresariales, referidos a las cuatro últimas.

Se trata además de variables altamente correlacionadas de forma interna, síntoma de la importante asociación interna entre las variables que integran cada uno de estos dos valores de reputación de los medios. Estos resultados refrendan la validez de constructo de ambos factores. 
Para finalizar respecto a las pruebas relativas a la medida de la reputación y a los mencionados factores o variables de reputación mediática, se ha analizado también la denominada fiabilidad, es decir, hasta qué punto los encuestados responderían aproximadamente del mismo modo a las cuestiones en distintos momentos del tiempo (Cronbach, 1970). Para ambos factores, los resultados obtenidos en el cálculo del alpha de Cronbach, instrumento usado habitualmente para este fin, superaron el 0,9, muy por encima del mínimo de 0,7 que se considera adecuado para un análisis como el que estamos realizando (Hair et al., 1999). De este modo, podemos concluir que el instrumento de medida utilizado también cumple con las condiciones de fiabilidad. Este hecho, unido a la mencionada validez de constructo que concluimos como resultado del análisis de componentes principales, permite no rechazar la primera de las hipótesis planteadas.

El segundo objetivo de este trabajo es determinar la influencia de ambos valores canónicos sobre la reputación de un medio de comunicación, desde la perspectiva de los profesionales de la comunicación. Para ello se ha realizado un análisis de regresión, en el que la variable ${ }^{2}$ dependiente es dicha reputación mediática, mientras que las variables independientes, que tratan de explicarla, son precisamente los dos valores canónicos obtenidos del análisis previo.

Los resultados no permiten rechazar la segunda hipótesis planteada: la reputación de un medio de comunicación se explica en muy buena medida a partir de sus valores periodísticos o profesionales y de sus valores empresariales. Como podemos ver en la tabla 5, entre ambos factores consiguen explicar el 72,1\% de la reputación de un medio, con una significación inferior a 0,001. Si únicamente tenemos en cuenta cada uno de los factores de forma aislada, podemos comprobar cómo los valores periodísticos o profesionales son los que tienen una mayor capacidad explicativa de la reputación (53,5\%), mientras que los factores empresariales explicarían el $18,5 \%$. De este modo, tampoco es posible refutar la tercera de las hipótesis.

Tabla 5. Regresión lineal sobre la medida directa de la reputación

\begin{tabular}{|l|c|c|c|}
\hline \multicolumn{1}{|c|}{ Modelo } & R & R cuadrado & R cuadrado ajustado \\
\hline Ambos factores & 0,850 & 0,722 & 0,721 \\
\hline Valores periodísticos y profesionales & 0,732 & 0,536 & 0,535 \\
\hline Valores empresariales & 0,432 & 0,186 & 0,185 \\
\hline
\end{tabular}

\section{Discusión y conclusiones}

Como hemos podido comprobar, los resultados que derivan de los análisis cuantitativos no permiten rechazar las hipótesis planteadas. Como se ha comentado a lo largo del texto, este estudio se basa en la aportación previa de Ortiz-de-Guinea-Ayala, Villafañe y Caffarel-Serra (2018), quienes construyen el fundamento inicial de la Teoría de la reputación aplicada al campo concreto de los medios de comunicación. Esta aportación, que supone una novedad, requiere de estudios empíricos que contrasten su validez. El presente estudio es un primer paso y permite constatar la idea de que la reputación mediática depende de dos valores canónicos -los periodísticos o profesionales y los valores empresariales que explican, según el estudio de regresión efectuado, más del 72\% de dicha reputación-y que además ambos valores distan mucho de ser equipotentes, ya que la cifra anterior comprende el 53,5\% correspondiente a los valores profesionales o periodísticos y el 18,5\% que suponen los valores empresariales. En consecuencia, la mejora o el deterioro de la reputación de un medio dependerán de ambos valores canónicos y de las variables que los definen, lo que debería proporcionar a los directivos y profesionales de los medios de comunicación una guía de actuación para trabajar en un ámbito tan relevante como es la reputación.

Son muy escasos los estudios empíricos de reputación sectorial referidos a otras industrias o sectores empresariales que sirvieran como referencia para comparar la importancia en la reputación de las prácticas profesionales frente a las empresariales. A pesar de ello, se hace difícil no pensar en la gran importancia de ese primer factor -la praxis profesional y periodística- que en base 100 supone las tres cuartas partes del peso de ésta en la reputación de los medios de comunicación frente a una cuarta parte que supone su devenir empresarial y su gestión. Este constituye un hallazgo de notable relevancia.

Como líneas futuras de investigación, sin ánimo de exhaustividad, se contempla la extensión del contraste de la existencia de esos valores canónicos para los diferentes grupos de interés que influyen en los medios de comunicación. La realización de una encuesta a población general es uno de los siguientes pasos naturales para el avance de esta línea. Por otro lado, también se está trabajando en el estudio de la traslación de la acción en reputación, esto es, cuáles son las actuaciones objetivas que influyen en los diferentes constructos de los que dependen la reputación de los medios de comunicación. Finalmente, resulta interesante la consideración de cuáles son los efectos concretos de esa mayor o menor reputación sobre los diferentes medios. Todas estas cuestiones, la gran mayoría sin resolver, contribuirán al asentamiento de esa Teoría de la reputación para los medios de comunicación. 


\section{Nota}

1. Aunque existen diferentes métodos de extracción de factores, el empleado en este estudio (componentes principales) opera directamente sobre los autovalores y autovectores de la matriz de correlaciones entre las distintas variables, mientras que otras técnicas actúan sobre transformaciones de dicha matriz.

2. El término variable se utiliza aquí en sentido estadístico. Desde el punto de vista de la reputación mediática la noción de variable hace referencia a las características en las que se descompone un mismo valor de reputación de un medio de comunicación; tiene, en consecuencia, otra significación diferente. Las variables de reputación mediática se podrán, a su vez, descomponer en indicadores, es decir entidades de tercer nivel que, además de satisfacer otras condiciones de validez (Villafañe, 2014, p. 44) han de ser medibles.

\section{Referencias}

Arregle, Jean-Luc; Hitt, Michael A.; Sirmon, David G.; Very, Philippe. (2007). "The development of organizational social capital: Attributes of family firms". Journal of management studies, v. 44, n. 1, pp. 73-95.

https://doi.org/10.1111/j.1467-6486.2007.00665.x

Barnett, Michael L.; Jermier, John M.; Lafferty, Barbara A. (2006). "Corporate reputation: The definitional landscape". Corporate reputation review, v. 9, n. 1, pp. 26-38.

https://doi.org/10.1057/palgrave.crr.1550012

Bhattacharya, C. B.; Sen, Sankar (2004). "Doing better at doing good: When, why, and how consumers respond to corporate social initiatives". California management review, v. 47, n. 1, pp. 9-24.

https://doi.org/10.2307/41166284

Boyd, Brian K.; Bergh, Donald D.; Ketchen, David J. (2010). "Reconsidering the reputation-performance relationship: A resource-based view". Journal of management, v. 36, n. 3, pp. 588-609.

https://doi.org/10.1177/0149206308328507

Bromley, Dennis-Basil (1993). Reputation, image, and impression management. John Wiley \& Sons. ISBN: 9780 471938699

Carreras, Enrique; Alloza, Ángel; Carreras, Ana (2013). Corporate reputation. LID Editorial. ISBN: 9788483567975

Chun, Rosa (2005). "Corporate reputation: Meaning and measurement". International journal of management reviews, v. 7, n. 2, pp. 91-109.

https://doi.org/10.1111/j.1468-2370.2005.00109.x

Chun, Rosa; Davies, Gary (2006). "The influence of corporate character on customers and employees: Exploring similarities and differences". Journal of the Academy of Marketing Science, v. 34, n. 2, pp. 138-146.

https://doi.org/10.1177/0092070305284975

Cravens, Karen; Goad-Oliver, Elizabeth; Ramamoorti, Sridhar (2003). "The reputation index: Measuring and managing corporate reputation". European management journal, v. 21, n. 2, pp. 201-212.

https://doi.org/10.1016/S0263-2373(03)00015-X

Cronbach, Lee J. (1970). Essentials of psychological testing. Harper \& Row. ISBN: 0063561263

Davies, Gary; Chun, Rosa (2002). "Gaps between the internal and external perceptions of the corporate brand". Corporate reputation review, v. 5, n. 2-3, pp. 2-3.

https://doi.org/10.1057/palgrave.crr.1540171

De-la-Fuente-Sabaté, Juan-Manuel; Quevedo-de-Puente, Esther (2003). “The concept and measurement of corporate reputation: an application to Spanish financial intermediaries". Corporate reputation review, v. 5, n. 4, pp. 280-301.

https://doi.org/10.1057/palgrave.crr.1540180

Fischer, Eileen; Reuber, Rebecca (2007). "The good, the bad, and the unfamiliar: The challenges of reputation formation facing new firms". Entrepreneurship theory and practice, v. 31, n. 1, pp. 53-75.

https://doi.org/10.1111/j.1540-6520.2007.00163.x

Fombrun, Charles J. (1996). Reputation: Realizing value from the corporate image. Boston: Harvard Business School Press. ISBN: 0875846335

Gioia, Dennis A.; Thomas, James B. (1996). "Identity, image, and issue interpretation: Sensemaking during strategic change in academia". Administrative science quarterly, v. 41, n. 3, pp. 370-403.

https://doi.org/10.2307/2393936

Grant, Robert M. (2006). Dirección estratégica. conceptos, técnicas y aplicaciones (5a ed.). Madrid: Thomson-Civitas. ISBN: 9788447026586 
Gulati, Ranjay (1998). "Alliances and networks". Strategic management journal, v. 19, n. 4, pp. $293-317$. https://doi.org/10.1002/(SICI)1097-0266(199804)19:4<293::AID-SMJ982>3.0.CO;2-M

Hair, Joseph F.; Anderson, Rolph E.; Tatham, Ronald L.; Black, William C. (1999). Análisis multivariante. Prentice Hall Madrid. ISBN: 8483220350

Hall, Richard (1993). "A framework linking intangible resources and capabilities to sustainable competitive advantage". Strategic management journal, v. 14, n. 8, pp. 607-618.

https://www.jstor.org/stable/2486860

Jensen, Michael C.; Meckling, William H. (1976). "Theory of the firm: Managerial behavior, agency costs and ownership structure". Journal of financial economics, v. 3, n. 4, pp. 305-360.

https://doi.org/10.1016/0304-405X(76)90026-X

Lange, Donald; Lee, Peggy M.; Dai, Ye (2011). “Organizational reputation: A review”. Journal of management, v. 37, n. 1, pp. 153-184.

https://doi.org/10.1177/0149206310390963

Martín-de-Castro, Gregorio; Navas-López, José-Emilio; López-Sáez, Pedro (2006). “Business and social reputation: Exploring the concept and main dimensions of corporate reputation". Journal of business ethics, v. 63, n. 4, pp. 361. https://doi.org/10.1007/s10551-005-3244-z

Morales-Blanco-Steger, Begoña; Fuente-Cobo, Carmen (2018). "Confianza de las audiencias en las marcas televisivas: propuesta de indicadores de responsabilidad social y reputación corporativa". El profesional de la información, v. 27, n. 3, pp. 537-547.

https://doi.org/10.3145/epi.2018.may.07

Nahapiet, Janine; Ghoshal, Sumantra (1998). "Social capital, intellectual capital, and the organizational advantage". Academy of management review, v. 23, n. 2, pp. 242-266.

https://doi.org/10.2307/259373

Ortiz-de-Guinea-Ayala, Yolanda; Villafañe, Justo; Caffarel-Serra, Carmen (2018). "Investigación para la evaluación de la reputación de los medios de comunicación”. Revista latina de comunicación social, n. 73, pp. 845-869. https://doi.org/10.4185/RLCS-2018-1285

Reuber, A. Rebecca; Fischer, Eileen (2005). "The company you keep: How young firms in different competitive contexts signal reputation through their customers". Entrepreneurship theory and practice, v. 29, n. 1, pp. 57-78. https://doi.org/10.1111/j.1540-6520.2005.00069.x

Rindova, Violina P.; Williamson, lan O.; Petkova, Antoaneta P.; Sever, Joy-Marie (2005). "Being good or being known: An empirical examination of the dimensions, antecedents, and consequences of organizational reputation". Academy of Management journal, v. 48, n. 6, pp. 1033-1049.

https://doi.org/10.5465/amj.2005.19573108

Shamsie, Jamal (2003). "The context of dominance: an industry-driven framework for exploiting reputation". Strategic management journal, v. 24, n. 3, pp. 199-215.

https://doi.org/10.1002/smj.291

Villafañe, Justo (2013). La buena empresa. Pearson Educación. ISBN: 9788490353066

Villafañe, Justo (2017). "Claves empíricas de la satisfacción y del compromiso del talento en las organizaciones". El profesional de la información, v. 26, n. 6, pp. 1159-1170.

https://doi.org/10.3145/epi.2017.nov.15

Williamson, Oliver E. (1975). Markets and hierarchies. New York. ISBN: 9780029353608

\section{Anexo. Cuestionario utilizado en el estudio cuantitativo}

\section{Ficha metodológica del estudio}

- Ámbito: nacional.

- Universo: periodistas.

- Tipo de entrevista: encuesta telefónica.

- Muestra: a realizar 135 entrevistas.

- Base de datos: listados de teléfonos de periodistas y Dircom.

- Distribución muestral: sin establecimiento de cuotas. 


\section{Reputación medios de comunicación}

P2. A nivel general, y sin referirse a un medio concreto, diferenciando entre prensa a papel, prensa digital, radio y televisión, ¿̇qué tipo de medio de comunicación le parece a usted...?

\begin{tabular}{|l|l|c|c|c|}
\cline { 3 - 5 } \multicolumn{2}{c|}{} & Prensa papel & Prensa digital & Televisión \\
\hline 0201 & Con más credibilidad & 1 & 2 & 4 \\
\hline 0202 & Con más rigor informativo & 1 & 2 & 3 \\
\hline 0203 & Más imparcial & 1 & 2 & 3 \\
\hline 0204 & Con más disposición a rectificar & 1 & 2 & 3 \\
\hline
\end{tabular}

P3. A continuación recogemos una serie de medios, dígame en qué medida conoce o sigue la información de cada uno de ellos.

\begin{tabular}{|c|c|c|c|c|c|}
\hline \multirow[b]{2}{*}{ Bloque 1} & & $\begin{array}{l}\text { Es un medio cuya } \\
\text { información sigo } \\
\text { habitualmente }\end{array}$ & $\begin{array}{c}\text { Es un medio que } \\
\text { sigo esporádica- } \\
\text { mente }\end{array}$ & $\begin{array}{l}\text { No sigo su informa- } \\
\text { ción, pero sí tengo } \\
\text { referencias }\end{array}$ & $\begin{array}{l}\text { No tengo práctica- } \\
\text { mente referencias } \\
\text { de este medio }\end{array}$ \\
\hline & \multicolumn{5}{|l|}{ Prensa } \\
\hline 0301 & $A B C$ & 1 & 2 & 3 & 4 \\
\hline 0302 & Elpaís & 1 & 2 & 3 & 4 \\
\hline 0303 & El mundo & 1 & 2 & 3 & 4 \\
\hline 0304 & La vanguardia & 1 & 2 & 3 & 4 \\
\hline 0305 & El periódico de Catalunya & 1 & 2 & 3 & 4 \\
\hline 0306 & El confidencial & 1 & 2 & 3 & 4 \\
\hline 0307 & Eldiario.es & 1 & 2 & 3 & 4 \\
\hline 0308 & Infolibre & 1 & 2 & 3 & 4 \\
\hline Bloque 2 & \multicolumn{5}{|l|}{ Radio } \\
\hline 0309 & COPE & 1 & 2 & 3 & 4 \\
\hline 0310 & Onda cero & 1 & 2 & 3 & 4 \\
\hline 0311 & $R N E$ & 1 & 2 & 3 & 4 \\
\hline 0312 & SER & 1 & 2 & 3 & 4 \\
\hline Bloque 3 & \multicolumn{5}{|l|}{ TV } \\
\hline 0313 & Antena 3 & 1 & 2 & 3 & 4 \\
\hline 0314 & La sexta & 1 & 2 & 3 & 4 \\
\hline 0315 & RTVE & 1 & 2 & 3 & 4 \\
\hline 0316 & Tele 5 & 1 & 2 & 3 & 4 \\
\hline
\end{tabular}

P4. Ahora nos vamos a centrar en algunos medios de los vistos anteriormente. Por favor, valore de 0 a 10 donde 0 es la peor valoración y 10 la mejor los siguientes aspectos para cada uno de los siguientes medios.

\begin{tabular}{|c|c|c|c|c|c|c|c|c|c|c|c|c|}
\hline & & 0 & 1 & 2 & 3 & 4 & 3 & 6 & $f$ & 8 & 9 & 10 \\
\hline \multicolumn{13}{|c|}{ Valores periodísticos o profesionales } \\
\hline 0401 & Credibilidad & 0 & 1 & 2 & 3 & 4 & 5 & 6 & 7 & 8 & 9 & 10 \\
\hline 0402 & Rigor informativo & 0 & 1 & 2 & 3 & 4 & 5 & 6 & 7 & 8 & 9 & 10 \\
\hline 0403 & Calidad periodística & 0 & 1 & 2 & 3 & 4 & 5 & 6 & 7 & 8 & 9 & 10 \\
\hline 0404 & Independencia & 0 & 1 & 2 & 3 & 4 & 5 & 6 & 7 & 8 & 9 & 10 \\
\hline 0405 & Ética y deontología profesional & 0 & 1 & 2 & 3 & 4 & 5 & 6 & 7 & 8 & 9 & 10 \\
\hline 0406 & Contribución a la democracia & 0 & 1 & 2 & 3 & 4 & 5 & 6 & 7 & 8 & 9 & 10 \\
\hline \multicolumn{13}{|c|}{ Valores empresariales } \\
\hline 0407 & Sostenibilidad económica & 0 & 1 & 2 & 3 & 4 & 5 & 6 & 7 & 8 & 9 & 10 \\
\hline 0408 & Transparencia en la gestión empresarial & 0 & 1 & 2 & 3 & 4 & 5 & 6 & 7 & 8 & 9 & 10 \\
\hline 0409 & Responsabilidad de la propiedad & 0 & 1 & 2 & 3 & 4 & 5 & 6 & 7 & 8 & 9 & 10 \\
\hline 0410 & Derechos y deberes de los trabajadores & 0 & 1 & 2 & 3 & 4 & 5 & 6 & 7 & 8 & 9 & 10 \\
\hline 0411 & Reputación global del medio & 0 & 1 & 2 & 3 & 4 & 5 & 6 & 7 & 8 & 9 & 10 \\
\hline
\end{tabular}

